\title{
Neutron beam line design of a white neutron source at CSNS
}

\author{
Hantao Jing ${ }^{1,2,5, \text { a }}$, Liying Zhang ${ }^{4,2,5}$, Jingyu Tang ${ }^{1,2}$, Xichao Ruan ${ }^{3}$, Changjun Ning ${ }^{1,2}$, Yongji Yu ${ }^{1,2}$, Pengcheng Wang ${ }^{1,2}$, \\ Qiang $\mathrm{Li}^{1,2}$, Jie Ren $^{3}$, Hongqing Tang ${ }^{3}$, and Xiangqi Wang ${ }^{4}$ \\ 1 Institute of High Energy Physics, Chinese Academy of Sciences (CAS), Beijing, China \\ 2 Dongguan Neutron Science Center, Dongguan, Guangdong, China \\ 3 Key Laboratory of Nuclear Data, China Institute of Atomic Energy, Beijing 102413, China \\ ${ }^{4}$ National Synchrotron Radiation Laboratory, University of Science and Technology of China, Anhui 230029, China \\ 5 State Key Laboratory of Intense Pulsed Radiation Simulation and Effect (Northwest Institute of Nuclear Technology), \\ Xi'an 710024, China
}

\begin{abstract}
China Spallation Neutron Source (CSNS), which is under construction, is a large scientific facility dedicated mainly for multi-disciplinary research on material characterization using neutron scattering techniques. The CSNS Phase-I accelerator will deliver a proton beam with an energy of $1.6 \mathrm{GeV}$ and a pulse repetition rate of $25 \mathrm{~Hz}$ to a tungsten target, and the beam power is $100 \mathrm{~kW}$. A white neutron source using the back-streaming neutrons through the incoming proton beam channel was proposed and is under construction. The back-streaming neutrons which are very intense and have good time structure are very suitable for nuclear data measurements. The white neutron source includes an 80-m neutron beam line, two experimental halls, and also six different types of spectrometers. The physics design of the beam line is presented in this paper, which includes beam optics and beam characterization simulations, with the emphasis on obtaining extremely low background. The first-batch experiments on nuclear data measurements are expected to be conducted in late 2017.
\end{abstract}

\section{Introduction}

China Spallation Neutron Source (CSNS) is a large scientific facility dedicated mainly for multidisciplinary research on material characterization using neutron scattering techniques $[1,2]$. The CSNS Phase-I, as shown in Fig. 1, is under construction and expected to be completed in early 2018. The CSNS accelerator complex is designed to deliver the proton beam with an energy of $1.6 \mathrm{GeV}$ and a pulse repetition rate of $25 \mathrm{~Hz}$ to a tungsten target, and the beam power at Phase-I is $100 \mathrm{~kW}$. The beam power will be upgraded to $500 \mathrm{~kW}$ at Phase-II. A white neutron source using the back-streaming neutrons through the incoming proton beam channel was proposed and is under construction $[3,4]$. The back-streaming neutrons that are modestly moderated by the cooling water passing through the target slices have a very wide energy spectrum (so-called white neutrons) and also a good time structure, and they are very suitable for nuclear data measurements.

\section{Layout of back-streaming white neutron source}

The back-streaming White Neutron Source (Back-n) at CSNS includes an 80-m neutron beam line (the first $20-\mathrm{m}$ beam line is common with the proton beam line) and two experimental halls. Two endstations with six detector systems (or spectrometers) in total with one in a time for nuclear data measurements are planned in order to carry out different types of experiments.

a e-mail: jinght@ihep.ac.cn
As shown in Fig. 2, the near endstation (ES\#1) at $56 \mathrm{~m}$ from the target is a high-flux experimental hall. The far endstation (ES\#2) at $76 \mathrm{~m}$ from the target is highresolution experimental hall. A preparation room is for preparing the experiments or maintaining the detector temporarily. Besides, there is also a local control room on the ground for remotely manipulating and monitoring the experiment process.

Thanks to the multiple apertures of the shutter (also functioning as a collimator) and two collimators, one can control the neutron beam intensity and beam spot sizes. According to the requirements of different experiments, one of the three beam spot settings as listed in Table 1 will be used. Due to space limitation, an in-room neutron dump has to be located at rear of ES\#2 [5]. The neutron beam window at about $26 \mathrm{~m}$ from the target can separate the different vacuum conditions between proton beam line $\left(10^{-6} \mathrm{~Pa}\right)$ and neutron beam line $\left(10^{-4} \mathrm{~Pa}\right)$. The appended motion system can control the $\mathrm{Cd}$ and/or $\mathrm{B}_{4} \mathrm{C}$ filters which absorb the low-energy neutrons to avoid the influence of low-energy neutrons on other repetition period.

\section{Physics design}

\subsection{Neutron energy spectrum and time resolution}

CSNS uses a sliced tungsten target, with water-cooling. The target size is $7 \mathrm{~cm}$ (Height) $\times 17 \mathrm{~cm}($ Width $) \times 65 \mathrm{~cm}$ (Length), including the water layers. The production, moderation, and transport of neutrons in the target have been simulated using FLUKA [6]. A proton beam window

(c) The Authors, published by EDP Sciences. This is an Open Access article distributed under the terms of the Creative Commons Attribution License 4.0 (http://creativecommons.org/licenses/by/4.0/). 


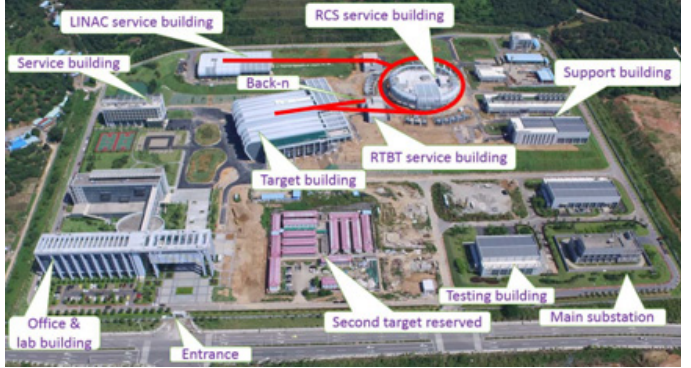

Figure 1. Bird's eye view of CSNS.

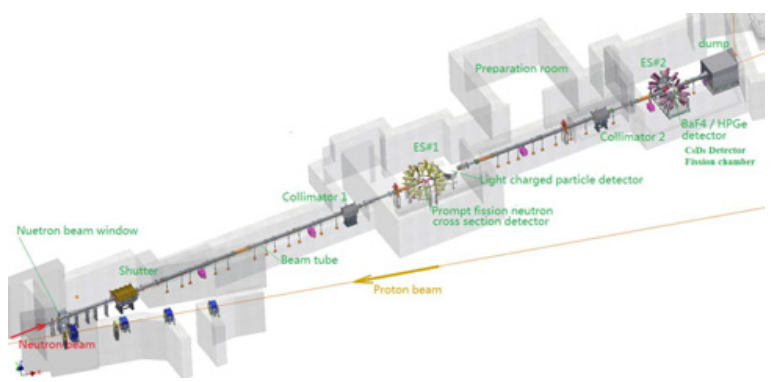

Figure 2. Layout of Back-n neutron beam line.

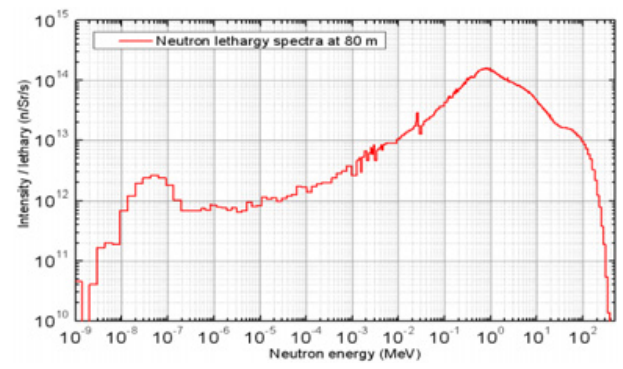

Figure 3. Back-n neutron energy spectrum at $80 \mathrm{~m}$.

(PBW) of about $1.5 \mathrm{~mm}$ in thickness and a neutron beam window (NBW) of $1 \mathrm{~mm}$ in thickness both in aluminium alloy are placed at $1.5 \mathrm{~m}$ and $26 \mathrm{~m}$ from the target respectively. The simulated back-streaming neutron spectrum and time resolution are shown in Figs. 3 and 4.

Different operation modes have been developed for the CSNS accelerator to provide neutron beams for different nuclear data measurements [7]. The smallest bunch width is $1.5 \mathrm{~ns}$ (rms). The transport of the back-streaming neutrons is simulated by FLUKA. In general, we can obtain the time resolution under $1 \%$ for the whole usable energy range ( $1 \mathrm{eV}$ to $100 \mathrm{MeV})$ with the combination of different operation modes ( $\mathrm{N}$-mode for normal neutron scattering, two D-modes for dedicated white neutron modes with beam power reduction). As shown in Fig. 4, for the D-Mode 2 the time resolution at Back- $n$ is comparable or even better with the CERN n-TOF above $100 \mathrm{keV}$.

\subsection{Neutron beam spots and fluxes}

According to the requirements of different experiments, three beam-spot settings are available in ES\#2. The shutter and collimators by using combined materials of iron and copper are designed to satisfy the beam sizes. On the shutter, five gears are configured. A no-hole position is used to block the neutron beam as a function of shutter. A tiny-hole position is used to lower the beam intensity to satisfy the low-flux white neutron experiments. The other

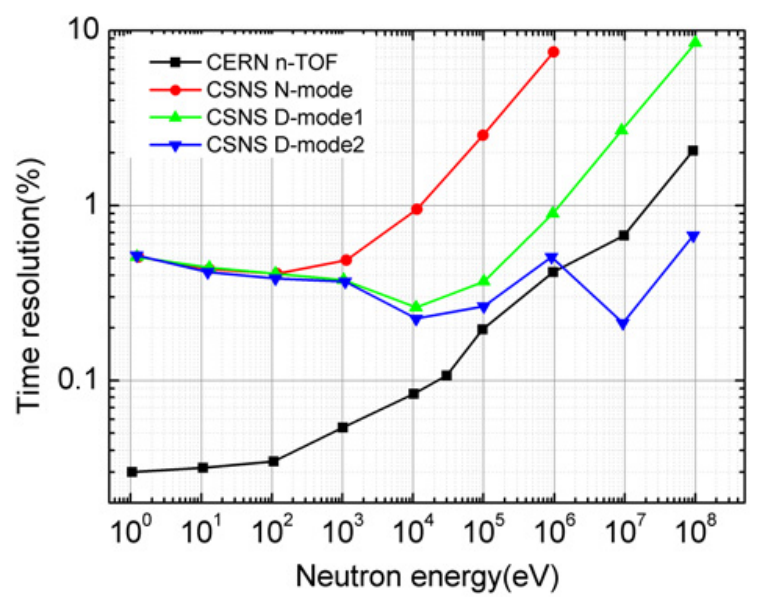

Figure 4. The simulated time resolution with different operation modes at $80 \mathrm{~m}$ vs that of CERN n-TOF.

three positions play a role of collimator to control the beam spots by aligning with Collimator \#1 and Collimator \#2. The collimation apertures and resultant fluxes for three cases are listed in Table 1. There is also a dedicated aperture set for neutron imaging, which will not be discussed here.

\subsection{Experimental backgrounds}

The experimental backgrounds include the gammas and scattering neutrons. For ES\#1, the neutron and gamma backgrounds include: 1) scattering in the inner wall of beam tube; 2) leaking through the front wall; 3) leaking through the lateral wall in which neutrons are produced by the proton beam loss on the nearby proton beam transport line. For the first, the beam tubes are designed to be of very large diameter of $300 \mathrm{~mm}$ and aluminium material is used. For the second, besides a thick concrete wall of $2 \mathrm{~m}$, filling the gap between the beam tube and the wall with iron sand and additional in-tube collimation by boroncontaining polythene bushings are also used. For the third, iron plates of $80 \mathrm{~cm}$ in thickness are put in the lateral wall to shield the neutrons from the proton beam line tunnel; in addition, a $5-\mathrm{cm}$ thick boron-containing polythene is attached to the inner surface of the hall except the ground to absorb neutrons. For ES\#2, besides the same sources of above 1) and 2) for ES\#1, the back-scattering neutrons and gammas from the dump which situates in the hall are also important. In order to suppress this effect, a sophisticated in-room dump has been designed [5]. The neutron and gamma backgrounds in the two endstations which have been simulated by FLUKA, are shown in Fig. 5. Another important background contribution from the experimental sample is difficult to suppress, but should be measured in details for case-to-case experiments.

\section{Radiation protection}

The shutter is a key component on the neutron beam line when one needs to switch the neutron beam off to enter in the experimental halls. The absorption block of the shutter is composed of combined 1.2-m iron and 0.3-m copper. As shown in Fig. 5, when the shutter is closed, the prompt radiation dose equivalent rate is much less than $1 \mu \mathrm{Sv} / \mathrm{h}$ in the ES\#1 even in the case of $500 \mathrm{~kW}$ beam power 
Table 1. Back-n neutron beam spots and corresponding fluxes.

\begin{tabular}{|c|c|c|c|c|c|c|c|}
\hline $\begin{array}{l}\text { ES2 } \\
\text { spot } \\
(\mathbf{m m})\end{array}$ & $\begin{array}{l}\text { Shutter } \\
(\mathrm{mm})\end{array}$ & $\begin{array}{c}\text { Coll\#1 } \\
(\mathbf{m m})\end{array}$ & $\begin{array}{c}\text { Coll\#2 } \\
(\mathbf{m m})\end{array}$ & $\begin{array}{l}\text { ES1 } \\
\text { spot } \\
(\mathbf{m m})\end{array}$ & $\begin{array}{c}\text { ES1 } \\
\text { flux } \\
\left(/ \mathrm{cm}^{2} / \mathrm{s}\right)\end{array}$ & $\begin{array}{c}\text { ES2 } \\
\text { flux } \\
\left(/ \mathrm{cm}^{2} / \mathbf{s}\right)\end{array}$ & $\begin{array}{c}\text { Possible } \\
\text { experiments }\end{array}$ \\
\hline$\varnothing 20$ & $\varnothing 3$ & $\varnothing 15$ & $\varnothing 40$ & $\varnothing 15$ & $1.27 \mathrm{E} 5$ & $4.58 \mathrm{E} 4$ & Low flux exp. \\
\hline$\emptyset 30$ & $\emptyset 12$ & $\varnothing 15$ & $\varnothing 40$ & $\varnothing 20$ & $2.20 \mathrm{E} 6$ & $7.81 \mathrm{E} 5$ & $(\mathrm{n}, \mathrm{t}),(\mathrm{n}, \gamma),\left(\mathrm{n}, \mathrm{n}^{\prime} \gamma\right),(\mathrm{n}, 2 \mathrm{n} \gamma)$ \\
\hline Ø60 & $\varnothing 50$ & $\varnothing 50$ & $\varnothing 58$ & $\varnothing 50$ & 4.33E7 & $1.36 \mathrm{E} 7$ & $(\mathrm{n}, \mathrm{f}),(\mathrm{n}, \mathrm{lcp})$, PFNS \\
\hline $90 \times 90$ & $78 \times 62$ & $76 \times 76$ & $90 \times 90$ & $75 \times 50$ & 5.98E7 & $2.18 \mathrm{E} 7$ & neutron radiography \\
\hline
\end{tabular}
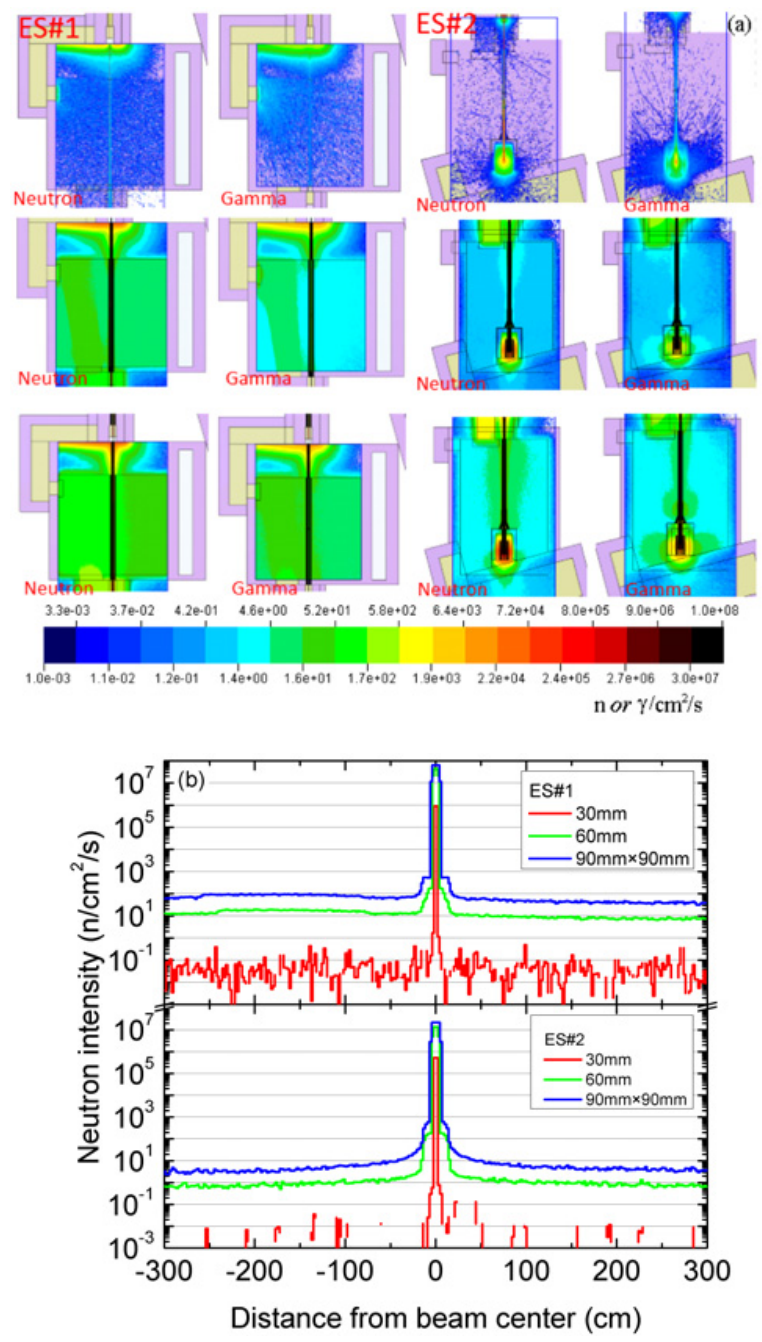

Figure 5. Neutron \& gamma fluences (a) and neutron profiles (b) in sample positions in ES\#1 (first three columns) and ES\#2 (last three columns).

(CSNS-II). It is safe for experimenters to enter the halls and carry out all necessary manipulations for experiments. When the beam is on target, the neutron beam line tunnel before ES\#1 is prohibited to enter.

When the neutron beam is on (shutter open), the prompt radiation dose equivalent rates in the back-n tunnel with a $100-\mathrm{kW}$ beam power for the three beam spot settings are still small, as shown in Fig. 6. The two red contours denote the dose values of $2.5 \mu \mathrm{Sv} / \mathrm{h}$ and $25 \mu \mathrm{Sv} / \mathrm{h}$. For strict safety, the person protection system (PPS) is designed to prohibit personnel to enter in the halls.

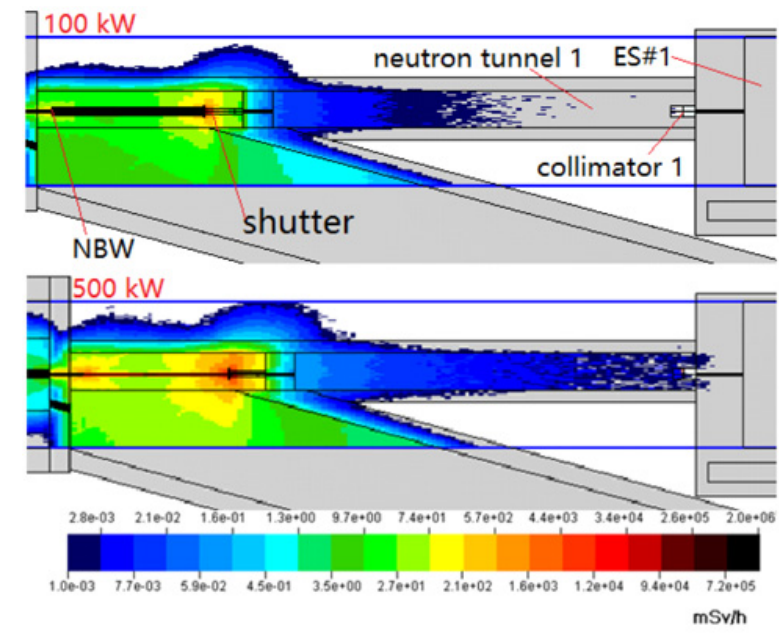

Figure 6. Prompt radiation dose equivalent rates in the Back$\mathrm{n}$ tunnel with the $100-\mathrm{kW}$ and $500-\mathrm{kW}$ beam power of proton accelerator when the neutron beam is switched off.

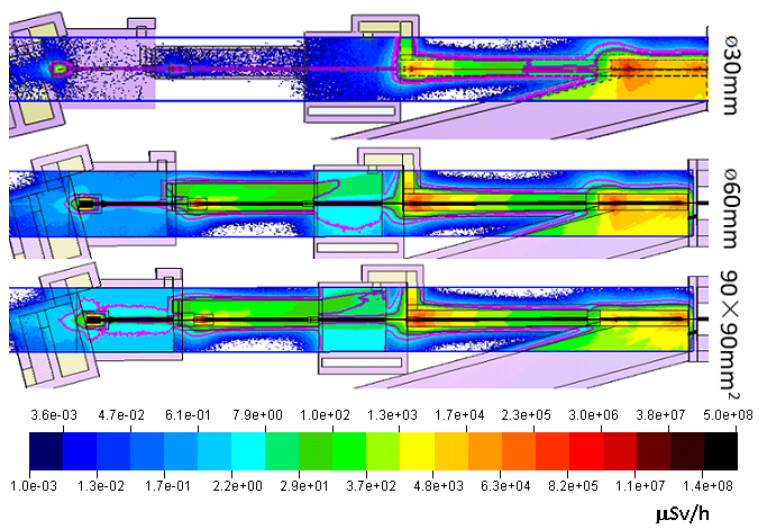

Figure 7. Prompt radiation dose equivalent rates in the Back-n tunnel with a $100-\mathrm{kW}$ beam power of proton accelerator for three beam spot settings.

\section{Conclusions and status}

The physics design for the back-streaming white neutron source at CSNS has been accomplished, including optics design, neutron beam profiles and neutron/gamma backgrounds with intense FLUKA simulations. The white neutron beams and the experimental conditions are proved to be excellent for nuclear data measurements.

At present, the main beam line components are under fabrication, and four detector systems are under development simultaneously. The first-batch experiments are expected to be conducted in late 2017, when the neutron target will receive the proton beam. 
The back-streaming white neutron source project is supported jointly by the CSNS project, the Key Research Program (project: 2016YFA0401601) of the Ministry of Science and Technology of China, the common fund by IHEP, CIAE, CAEP, NINT and USTC, and a fund (project: SKLIPR1517) from the State Key Laboratory of Intense Pulsed Radiation Simulation and Effect.

\section{References}

[1] S.X. Fang, et al., J. Kor. Phys. Soc. 48, 697 (2006)

[2] Hesheng Chen, Xunli wang, Nature Materials 15, 689 (2016)
[3] TANG Jing-Yu, FU Shi-Nian, JING Han-Tao, et al., Chinese Physics C 34(1), 121 (2010)

[4] H.T. Jing, J.Y. Tang et al., Nuclear Instruments and Methods in Physics Research A621, 91 (2010)

[5] L.Y. Zhang, H.T. Jing, J.Y. Tang, X.Q. Wang, Radiation Physics and Chemistry 127, 133 (2016)

[6] A. Ferrari, P.R. Sala, A. Fass o, J. Ranft, FLUKA: a multi-particle transport code, CERN 2005-10, INFN/TC-05/11, SLAC-R-773, 2005

[7] Ye Zou, Jingyu Tang et al., Phys. Rev. ST. accelerators and beams 17, 060101 (2014) 